East African Medical Journal Vol. 86 No. 4 April 2009

ANTIRETROVIRAL THERAPY DOSE ADJUSTMENTS BASED ON CALCULATED CREATININE CLEARANCE J. Shavadia, MBChB, Resident, The Aga Khan University Hospital Nairobi, P. O. Box 40374-00100, Nairobi, Kenya., G. Tesfaldet, MD, MMed, Consultant Physician and M. Twahir, MBChB, MMed, Cert Nephrology, Consultant Physician and Nephrologist, The Aga Khan University Hospital Nairobi, P. O. Box 40374-00100, Nairobi Kenya

Request for reprints to: Dr. J. Shavadia, The Aga Khan University Hospital, Nairobi, P. O. Box 40374-00100, Nairobi Kenya

\title{
ANTIRETROVIRAL THERAPY DOSE ADJUSTMENTS BASED ON CALCULATED CREATININE CLEARANCE
}

\author{
J. SHAVADIA, G. TESFALDET and M. TWAHIR
}

\begin{abstract}
Background: Whereas therapy for HIV is dependent on level of creatinine clearance, most laboratories locally only report an absolute creatinine value. There is likelihood that the patients already on antiretroviral therapy (ART) may have required dosage adjustment at the time of initiation of therapy or sometime during ongoing therapy. This paper explores a group of patients who are already on ART to determine their creatinine clearance and assess the need for ART dose adjustment.

Objective: To determine the proportion of stable HIV outpatients who have a documented creatinine clearance $(\mathrm{CrCI})$ and the proportion requiring antiretroviral drug dose adjustments depending on their creatinine clearance.

Design: Retrospective observational study.

Setting: One stop HIV medical clinic, Aga Khan University Hospital, Nairobi between January and February 2007.

Subjects: Ninety three patients seen.

Results: None of the study subjects had a calculated creatinine clearance in their medical records. Fifteen of the 93 patients $(16.1 \%)$ had no serum creatinine performed in the twelve months preceding the last clinic visit. Nine of the remaining 78 patients $(11.5 \%)$ had evidence of renal insufficiency $(\mathrm{CrCI}<60 \mathrm{mls} / \mathrm{min})$ as estimated by the Cockroft Gault method, with six patients $(7.7 \%)$ requiring dose adjustments to the one or more drugs in their antiretroviral therapy (ART) regime ( $\mathrm{CrCI}<50 \mathrm{mls} / \mathrm{min})$.

Conclusion: It is imperative to have a CrCI prior to and during follow up of patients with HIV disease on ART to reduce potential drug toxicities and interactions, especially with the increased utilisation of newer and potentially more nephrotoxic antiretrovirals.
\end{abstract}

\section{INTRODUCTION}

Although the Infectious Disease Society of America (IDSA) recommends measurement of renal function prior to initiation of antiretroviral therapy (ART) (1) and regular screening of renal chemistry while on ART, majority of the patients in resource constrained settings do not conform to these guidelines largely due to financial constraints. Those that attempt to measure renal chemistries, use serum creatinine as an estimation of the renal function. However, serum creatinine alone is not ideal for the measurement of renal function. Many HIV positive patients present late in our set-up. At presentation, these patients usually have weight loss with significant reduction in muscle mass. Serum creatinine is influenced by muscle mass even without a change in glomerular filtration rate. The current recommendation is that the glomerular filtration rate (GFR), rather than serum creatinine, be used to assess the degree of renal impairment and to follow the course of renal disease.

Several modalities have been utilised in the general population to determine the GFR. A twenty four hour urine collection and subsequent measurement of creatinine clearance $(\mathrm{CrCl})$ was once commonly used. However, attendant problems of accurate urine collection, timing, convenience and cost have relegated this method predominantly to in-patients where this can be supervised. It is now acceptable to estimate creatinine clearance using either Cockcroft Gault or the modification of diet in renal disease (MDRD) formula $(2,3)$. Based on this, most guidelines on ART recommend antiretroviral 
dose adjustment based on calculated GFR rather than serum creatinine levels (1-4).

Although highly active antiretroviral therapy (HAART) has revolutionised the treatment of HIV-infected patients, new problems have emerged, such as viral resistance, drug interactions and drug-related adverse effects. This has therefore made ARV drug prescription increasingly complex, predisposing to prescription errors, both in hospitalised patients and the out-patient setting (5-9). In a review of 209 admissions over a one-year period, Rastegar et al (7) reported that in $16.7 \%$ of hospitalised HIV-infected patients, ARV medication errors were due to incorrect amount or frequency of dosage; over half of these errors were attributable to failure to appropriately adjust dosage for renal insufficiency.

Several antiretroviral medications may have renal adverse events. Conversely, many patients with already impaired renal function have increased incidence of side effects of ART such as neuropathy, lipodystrophy and Fanconi syndrome (1). There have been several case reports of renal failure in patients on teno fovir (10-14), most of whom had normal renal function prior to initiation of ART. This deterioration of renal function is potentially worse in patients who have an impaired baseline creatinine clearance.

Wools-Kaloustian et al (15) performed a cross-sectional study in an ARV-naive Kenyan population and found baseline renal insufficiency defined as a $\mathrm{CrCI}<60 \mathrm{mls} / \mathrm{min}$ in $11.5 \%$ and $\mathrm{CrCl}$ $<50 \mathrm{mls} / \mathrm{min}$ in $4.8 \%$ of the study population. With an HIV prevalence rate of $7.8 \%$ (16) in the Kenyan population of 37 million, over two million would be HIV-infected of whom, close to $300,000 \mathrm{HIV}$ infected patients may have potential baseline renal insufficiency. When these patients are commenced on HAART, close monitoring of their renal function would be required, both prior to and during therapy.

Whereas therapy of HIV is dependent on level of creatinine clearance, most of the laboratories in our country only report creatinine as an absolute value. There is a likelihood that the patients already on ART may have required dosage adjustment at the time of initiation of therapy or sometime during ongoing therapy. This paper explores this group of patients who are already on ART and determine their creatinine clearance thereby determining those whose treatment should have been adjusted.

\section{MATERIALS AND METHODS}

One hundred and twenty seven patient records which met criteria for inclusion and excluded thirty four who either were not on ART or had insufficient data on their age, gender and weight.
Analysis was therefore performed for ninety-three patients' records between January and February 2007. Since these patients re-attended the outpatient clinic after February 2007, the last date of appointment in the year 2007 was noted and the following parameters obtained: Age, gender and race; weight at the last appointment date; outpatient serum creatinine in the preceding 12 months of the last review date. We only utilised serum creatinine done as an out-patient since this parameter may change when patients are acutely unwell; ART regime and dosing as per the last review date; last available CD (4) count and plasma viral load within the preceding $12 \mathrm{mo}$.

Every eligible patient had a serum creatinine clearance calculated using both the Cockcroft-Gault and simplified MDRD method. Dosing recommendations was utilised as per the guidelines for the use of antiretroviral agents in HIV -infected adults and adolescents (15).

\section{RESULTS}

The mean age was 42.8 years ( 25 - 67 years) and males comprised $61.3 \%$ of the study population giving a male: female ratio of 1.6: 1. All our patients were black Africans.

Figure 1

ART use was distributed as follows

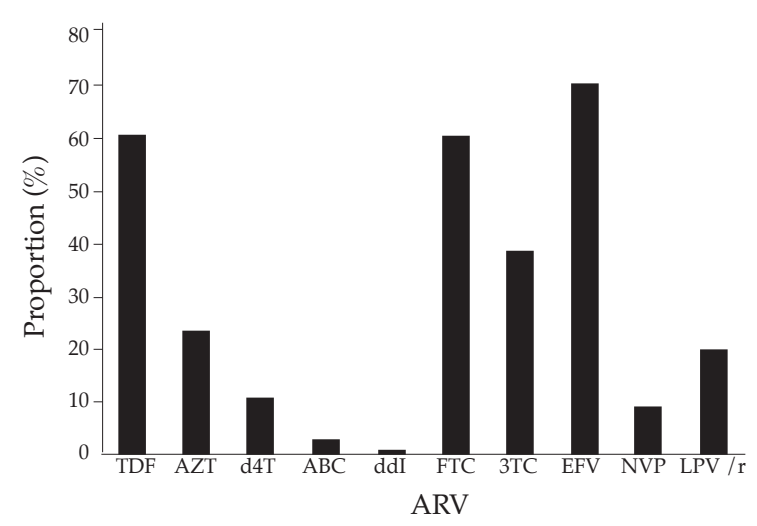

$\mathrm{TDF}=$ Tenofovir

$\mathrm{AZT}=$ Zidovudine

$\mathrm{d} 4 \mathrm{~T}=$ Stavudine

FTC $=$ Emtricitabine

3TC $=$ Lamivudine

$\mathrm{ABC}=$ Abacavir

$\mathrm{EFV}=$ Efavirenz

$\mathrm{NVP}=$ Nevirapine

ddI $=$ Didanosine

LPV / $\mathrm{r}=$ Lopinavir /

Ritonavir

Seventy eight point two per cent of patients had $\mathrm{CD}_{4}$ counts $\geq 200$ with the mean $\mathrm{CD}_{4}$ count of 385 cells $/ \mathrm{mm}^{3}$ (2-1304 cells $\left./ \mathrm{mm}^{3}\right)$. On analysis of the plasma viral load data available for $85(91.4 \%)$ patients, $63(74.1 \%)$ patients had undetectable viral loads ( $<50$ copies $/ \mathrm{ml})$.

None of the study patients either had a calculated creatinine clearance recorded in 
their medical records or had had ART dosages adjusted. Fifteen of the 93 patients evaluated (16.1\%) had not had a serum creatinine done in the 12 months preceding the last clinic visit. Of the 78 patients who had data on their serum creatinine, nine $(11.5 \%)$ had a $\mathrm{CrCl}<60 \mathrm{mls} / \mathrm{min}$ and six $(7.7 \%)$ had a $\mathrm{CrCI}<50 \mathrm{mIs} / \mathrm{min}$ as per the Cockroft Gault method while 7.6\% and 3.8\% had $\mathrm{CrCI}<60 \mathrm{mls} / \mathrm{min}$ and $<50 \mathrm{mls} / \mathrm{min}$ respectively, as per the simplified MDRD formula.

\section{DISCUSSION}

None of the study patients had a documented calculated creatinine clearance on which their ART dosing was based on. This is not in keeping with the recommendations either from the guidelines for the use of antiretroviral agents in HIV-infected adults and adolescents (17) or the IDSA guidelines (1) which recommend ART dosing based on the CrCI. It is also not in tandem with the WHO guidelines (18) for ARV monitoring in regional referral centres where full serum chemistries have been advocated as opposed to resource-limited settings at primary health care and district levels.

From our study cohort, we also found $7.7 \%$ of the patients had a $\mathrm{CrCI}<50 \mathrm{mls} / \mathrm{min}$, reflecting the proportion of patients who require dose adjustment in their ART, mainly in the nucleoside and nucleotide reverse transcriptase inhibitor dosing. Although not our primary aim, it would have been interesting to identify the proportion and nature of adverse events in this cohort of patients who actually had renal insufficiency and required ART dose adjustment. The predominant agents requiring dose modification from our study included: tenofovir, emtricitabine, stavudine and lamivudine. An additional $3.8 \%$ of the study patients had a $\mathrm{CrCI}$ between $51-60 \mathrm{mls} / \mathrm{min}$ and will therefore require close monitoring of their renal function. The need for dose adjustment of ART in patients with chronic kidney disease is under-appreciated by physicians offering care to patients with HIV disease.

Over $60 \%$ of the patients in our study were on a tenofovir based ARV regime. However, the guidelines for antiretroviral therapy in Kenya (19) recommend the use of either stavudine or zidovudine as the nucleoside backbone. The difference is likely to be due to the simplicity of use and safety profile of tenofovir, hence physician and patient preferences in using this drug.

With increasing use of potentially nephrotoxic antiretrovirals, this calls for physicians taking care of patients with HIV disease to be vigilant in monitoring renal function and calculate the $\mathrm{CrCI}$ both prior and during treatment. It also adds to the growing momentum that $\mathrm{CrCI}$ rather than absolute creatinine values be reported by all laboratories to facilitate drug dosing and reduce toxicity profiles due to inadvertent drug overdosing.

The IDSA guideline recommends use of the Cockroft-Gault formula for calculation of the $\mathrm{CrCI}$ to base drug dosing on. In our study, the Cockroft-Gault formula was able to identify twice as many patients with an estimated $\mathrm{CrCI}<50 \mathrm{mls}$ / min, compared to the modified MDRD method. Due to this superiority and the ease of calculation of the CrCI using the Cockroft-Gault equation, this should probably be the recommended method in our population. However, this formula has neither been validated in our population nor in patients with HIV disease. Therefore, more local and regional data comparing the calculated methods of creatinine clearance to urinary clearance of exogenous filtration markers is required in patients with HIV disease.

Although not a primary goal of our study, we also noted that none of our patients had routine urinalysis done to test for proteinuria. Proteinuria is one of the presentations of HIV related kidney disease. This would have provided further supporting evidence to identify patients with kidney injury.

In keeping with our study, available local and regional evidence suggest that approximately $11.5 \%$ of ARV-naive HIV infected patients have baseline renal insufficiency ( $\mathrm{CrCI}<60 \mathrm{mls} / \mathrm{min}$ ) (15). However, our study demonstrated a higher proportion of patients $7.7 \%$ versus $4.8 \%$ (5) with hitherto undetected significant renal dysfunction of $\mathrm{CrCI}<50 \mathrm{ml} / \mathrm{min}$ requiring dosage adjustment of the ARV prescription. This implies these patients are exposed to higher drug toxicity and nephrotoxic effects of the medication.

In conclusion, the results of this study provides regional evidence, in addition to other locoregional studies (12), on the estimates of HIV infected patients requiring adjustments to their $\mathrm{ARV}$ regime based on their $\mathrm{CrCI}$. It also cautions all treating physicians to calculate an estimated GFR both prior to, and after initiation of ART to ensure appropriate antiretroviral dosage and hence prevent attendant ARV toxicity. In addition, it calls for laboratories to report $\mathrm{CrCI}$ using the Cockroft Gault method rather than absolute creatinine values to facilitate improvement in care for patients with HIV disease.

\section{ACKNOWLEDGEMENT}

To Dr. J.O. Jowi for his valuable critique of the paper. 


\section{REFERENCES}

1. Gupta, S. K., Eustace, J.A., Winston, J.A. et al. Guidelines for the management of chronic kidney disease in HIV -infected patients: recommendations of the HIV Medicine Association of the Infectious Diseases Society of America. Clin. Infect. Dis. 2005; 40: 1559-1585.

2. Cockcroft, D.W. and Gault, M. H. Prediction of creatinine clearance from serum creatinine. Nephron. 1976; 16: 31-41.

3. Levey, A. S., Bosch, J. P., Lewis, J.B. et al. A more accurate method to estimate glomerular filtration rate from serum creatinine: a new prediction equation. Modification of Diet in Renal Disease Study Group. Ann. Intern. Med. 1999; 130: 461-470.

4. Duggan, J. M., Sahloff, E.G. and Moudgal, V.V. Use of highly active antiretroviral therapy in patients with renal insufficiency. Pharmacotherapy. 2005; 5: 698-708.

5. Purdy, B. D., Raymond, A. M. and Lesar, T. S. Antiretroviral prescribing errors in hospitalized patients. Ann. Pharmacother. 2000; 34: 833-838.

6. DeLorenze, G. N., Follansbee, S. F., Nguyen, D. P., et al. Medication error in care of HIV / AIDS patients: electronic surveillance, confirmation and adverse events. Med. Care. 2005; 43: 63-68.

7. Rastegar, D. A., Knight, A. M. and Monolakis, J. S. Antiretroviral medication errors among hospitalized patients with HIV infection. Clin. Infect. Dis. 2006; 43: 933-938.

8. Tourret, J., Tostivint, I., Tezenas, Du Montcel, S., et al. Antiretroviral drug dosing errors in HIV infected patients undergoing hemodialysis. Clin. Infect. Dis. 2007; 45: 779-784.

9. Willig, J.H., Westfall, A.O., Allison, J. et al. Nucleoside reverse-transcriptase inhibitor dosing errors in an outpatient HIV clinic in the electronic medical record era. Clin. Infect. Dis. 2007; 45: 658-661.

10. Patel, S. M., Zembower, T. R., Palella, F., et al. Early onset of tenofovir-induced renal failure: case report and review of the literature. Scientific World J. 2007; 7: 1140-1148.

11. Krummel, T., Parvez-Braun, L., Frantzen, L. et al. Tenofovir-induced acute renal failure in an HIV patient with normal renal function. Nephrol Dial Transplant. 2005; 2: 473-474.

12. Callens, S., De Roo, A. and Colebunders, R. Fanconilike syndrome and rhabdomyolysis in a person with HIV infection on highly active antiretroviral treatment including tenofovir. J. Infect. 2003; 47: 262-263.

13. Karras, A., Lafaurie, M., Furco, A. et al. Tenofovir related nephrotoxicity in human immunodeficiency virus-infected patients: three cases of renal failure, Fanconi syndrome and nephrogenic diabetes insipidus. Clin. Infect. Dis. 2003; 36: 1070-1073.

14. Creput, C., Gonzalez-Canali, G., Hill, G. et al. Renal lesions in HIV-l-positive patient treated with tenofovir. AIDS. 2003; 17: 935-937.

15. Wools-Kaloustian, K., Gupta, S. K., Muloma, E. et al. Renal disease in an antiretroviralnaive HIVinfected outpatient population in Westem Kenya. Nephrol Dial Transplant. 2007; 8: 2208-2212.

16. Kenya Aids Indicator Survey 2008.

17. Guidelines for the Use of Antiretroviral Agents in HIV -infected Adults and Adolescents Jan 29, 2008.

18. Scaling Up Antiretroviral Therapy In ResourceLimited Settings; Treatment Guidelines For A Public Health Approach 2003 revision WHO Geneva 2004.

19. Guidelines for antiretroviral drug therapy in Kenya, $3^{\text {th }}$ edition Dec 2005. 